Int. J. Electrochem. Sci., 13 (2018) $8867-8888$

\title{
Anodization of aluminum in highly viscous phosphoric acid PART 1: Investigation of anodic oxide layers by scanning electron microscopy (SEM) and in-situ electrochemical impedance spectroscopy (in-situ EIS)
}

\author{
Lissy Berndt ${ }^{1,2}$, Malte Kleemeier ${ }^{1}$, Karsten Thiel ${ }^{1}$, Andreas Hartwig ${ }^{1,2}$, Malte Burchardt ${ }^{1, *}$ \\ ${ }^{1}$ Fraunhofer Institute for Manufacturing Technology and Advanced Materials IFAM, Wiener Straße \\ 12, 28359 Bremen, Germany \\ ${ }^{2}$ University of Bremen, Chemical Department, Leobener Str., 28359 Bremen, Germany \\ *E-mail: malte.burchardt@ifam.fraunhofer.de
}

doi: 10.20964/2018.09.11

Received: 23 March 2018 / Accepted: 29 May 2018 / Published: 5 August 2018

\begin{abstract}
A newly developed self-sticking tape which can be removed without residue facilitates local anodization on aluminum in cases of repair or re-work. The most prominent difference compared to conventional bath anodization is the exceptionally high viscosity of the electrolyte employed in the anodization tape. The impact of the highly viscous electrolyte on anodization is not yet known and was therefore investigated by SEM and in-situ electrochemical impedance spectroscopy (in-situ EIS). By employing cryo-SEM it could be shown that at the late stages of tape anodization ( 25 min at $25 \mathrm{~V}$ ) products of the anodization process accumulate near the anodized surface and partly block it. According to XPS analysis, the blocking layer mainly consists of organic material originating from the tape adhesive, while the concentration of aluminium ions is relatively low. The high frequency part of the in-situ EIS was evaluated with respect to the electrical properties which commonly characterize the barrier layer of the anodic oxide, namely its resistance and capacitance. At the beginning, the values for tape anodization are in good agreement with the data for a conventional bath anodization process. However, large deviations were found for longer anodization times. These deviations could also be attributed to a partial blocking of the anodic oxide. Using a model originally proposed by Jütner and Lorenz [12], the degree of coverage could be precisely determined from the in-situ EIS data. A good agreement with the coverage data from cryo-SEM was found.
\end{abstract}

Keywords: Anodization tape, porous anodic oxide, viscous electrolyte, SEM, in-situ electrochemical impedance spectroscopy

$\underline{\text { FULL TEXT }}$ 
Int. J. Electrochem. Sci., Vol. 13, 2018

(C) 2018 The Authors. Published by ESG (www.electrochemsci.org). This article is an open access article distributed under the terms and conditions of the Creative Commons Attribution license (http://creativecommons.org/licenses/by/4.0/). 